\title{
Flavoromics approach to identifying the key aroma compounds in traditional Chinese milk fan
}

\author{
Huaixiang Tian, Xiaolin $\mathrm{Xu}$, Chen Chen, and Haiyan $\mathrm{Yu}^{*}$ \\ Department of Food Science and Technology, Shanghai Institute of Technology, Shanghai 201418, China
}

\begin{abstract}
To determine the key aroma compounds in Chinese milk fan cheese, samples from 6 of the most popular handmade milk fan workshops in Yunnan province of China were analyzed by gas chromatography-mass spectrometry (GC-MS), gas chromatography-olfactometry (GC-O), sensory evaluation, aroma recombination and omission experiments. Seventy-one aroma compounds were identified by GC-MS, and 31 odor-active compounds were detected by GC-O. The relationships between the 31 odor-active compounds and 10 sensory evaluation properties were explored by partial least squares discriminant analysis. The results identified 23 initial key aroma compounds, which were used to simulate the aroma profile of milk fan in aroma recombination experiments. Aroma omission experiments identified propanoic acid, butanoic acid, octanoic acid, octanal, nonanal, 2-nonanone, and ethyl hexanoate as the key aroma compounds. Propanoic acid and butanoic acid contributed to cheese and rancid aromas, octanal and nonanal to the fat aroma, octanoic acid and 2 -nonanone to the cheese aroma, and ethyl hexanoate to the fruity aroma of milk fan.
\end{abstract}

Key words: milk fan, key aroma compounds, gas chromatography-mass spectrometry, gas chromatography-olfactometry, aroma recombination and omission

\section{INTRODUCTION}

Milk fan is a traditional Chinese cheese that has been produced for more than one thousand years, and it is a famous delicacy of the Bai ethnic minority in Yunnan Province (Liu et al., 2009). Named after its characteristic paper fan shape, milk fan is about 30 to $40 \mathrm{~cm}$ long, 8 to $10 \mathrm{~cm}$ wide, and $<2 \mathrm{~mm}$ thick (Liu et al., 2011). It is made from the milk of the Dengchuan yak, a breed of dairy cattle in southern China (Ma et al., 2012). At

Received April 13, 2019

Accepted July 11, 2019.

*Corresponding author: hyyu@sit.edu.cn an altitude of about $1,900 \mathrm{~m}$, the Dengchuan region has a temperate climate throughout the year with plentiful rain, which increases the types and quantities of microorganisms. The processing technology of milk fan differs from that of curd-coagulated cheese. Milk fan is a traditional acid-curd cheese made by adding papaya acid juice to raw milk (which alters the $\mathrm{pH}$ value), curding, blanching, stretching, forming, and drying (Zhang et al., 2014). The unique raw materials and processing technology give milk fan its unique flavor.

The flavor compounds in cheese have been extensively investigated, with studies having identified more than 3,000 volatile and nonvolatile components (Fox and Wallace, 1997). The aroma characteristics of cheese are mainly provided by the volatile components, including fatty acids, alcohols, aldehydes, esters, ketones, lactones, pyrazines, sulfur compounds, amines, and other flavor-active compounds (Masotti et al., 2017; Pelin et al., 2018). However, not all flavor compounds can be perceived by human olfactory receptors and thus contribute to the aroma characteristics (Bertuzzi et al., 2018).

The key aroma compounds can produce the perception of aromas in the human brain through interaction with olfactory organs (Dunkel et al., 2014). In recent years, the flavoromics approach to food flavor analysis has emerged to identify key aroma compounds in food (Gracka et al., 2016). The approach uses high-resolution instruments to collect sample data, which are then analyzed in combination with stoichiometric methods to fit the artificial sensory evaluation data (Karametsi et al., 2014). The gas chromatography-olfactory (GC-O) method combines instrument data with sensory data to provide a valuable tool for analyzing odor-active compounds and studying the activity of key aroma compounds (Song and Liu, 2018). The GC-O method has been used to investigate the odor-active compounds of milk (Jo et al., 2018b), beverages (Zhao et al., 2018), and other foods (Zhu and Xiao, 2018). Majcher et al. (2014) and Jo et al. (2018a) identified the odor-active compounds in cheese using GC-O. Stoichiometric methods have often been used to analyze large amounts of data to reveal hidden information (Medvedev et al., 
2017). For example, the relationships between the relative abundance of odor-active compounds in cheese and their sensory properties were investigated by partial least squares regression (PLSR; Zabaleta et al., 2016). Zhang et al. (2018) analyzed the relationship between sensory indicators and GC-O data using PLSR and found that some substances with low content, such as methyl 2-methyl-3-furanyl disulfide, had significant effects on the flavor characteristics of semi-hard cheeses (Zhang et al., 2018).

Aroma recombination and omission experiments can be used to verify results of key detected aroma compounds and the contribution of such compounds to the overall aroma. Majcher et al. (2018) verified that methyl ketones were not key aroma compounds in Lazur cheese by aroma recombination and addition experiments. Gao et al. (2014) determined that $\beta$-damascenone and ethyl acetate were the key aroma compounds in the typical pleasant, fruity, and floral aromas of Chinese liquor by means of GC-O, odor activity values (OAV), and omission experiments. However, there have been few investigations of the key aroma compounds in milk fan.

The main aim of this study was to identify the key aroma compounds in Chinese traditional milk fan using a flavoromics approach. A flowchart of the experimental process is presented in Figure 1. Sensory evaluation was used to describe the sensory characteristics of milk fan. The aroma components were analyzed by GC-MS, and the odor-active components were identified by GCO. Then, partial least squares discriminant analysis (PLS-DA) was used to assess correlations between the odor-active compounds and sensory properties to obtain the initial key aroma compounds. Finally, aroma recombination and omission experiments were used to further confirm the key aroma compounds and their contributions to the sensory characteristics. The results can be used as a reference for the quality evaluation, further promotion, and development of Chinese traditional milk fan.

\section{MATERIALS AND METHODS}

\section{Samples}

Six milk fan samples (Y1, Y2, Y3, Y4, Y5, and Y6) made from the same materials and using the same processing techniques were purchased from 6 of the most popular handmade workshops in Yunnan Province, China. Although the processing was almost identical, specific parameters, such as $\mathrm{pH}$ of the acid juice and curd temperature and time, were different. The samples were transported to the laboratory in ice boxes the day after production (March 14, 2018) and frozen at $-20^{\circ} \mathrm{C}$ until they were analyzed.

\section{Chemicals}

Propionic acid, butanoic acid, 3-methyl-butyric acid, pentanoic acid, hexanoic acid, heptanoic acid, octanoic acid, decanoic acid , 3-methylbutyraldehyde, octanal, nonanal, (E)-2-octenal aldehyde, 1-pentanol, 1-hexanol, 1,6-octadiene-3-alcohol, nonanol, para-cresol, 2-heptanone, 3-octanone, 2-nononone, acetophenone, ethyl butyrate, ethyl caproate, ethyl octanate, and D-limonene were purchased from Sigma-Aldrich (St. Louis, MO). 2-Octanol (internal standard, IS) and $n$-alkane standards $\left(\mathrm{C}_{6}-\mathrm{C}_{30}\right)$ were purchased from Dr. Ehrenstorfer GmbH Co. Ltd., Germany. All of the chemicals were of chromatographic grade.

\section{Solid-Phase Microextraction of Aroma Compounds in Milk Fan}

The milk fan samples (4 g) were thawed for $24 \mathrm{~h}$ at $4^{\circ} \mathrm{C}$, kept at room temperature for $1 \mathrm{~h}$, and then cut evenly into cubes. The cube samples and $100 \mu \mathrm{L}$ of IS solution $(13 \mathrm{mg} / \mathrm{L})$ were placed in a $15-\mathrm{mL}$ vial and covered with Teflon covers. The aroma compounds were extracted by using a solid-phase microextraction (SPME) fiber (Supelco Inc., Bellefonte, PA). The main parameters for SPME analysis were optimized based on the method of Jo et al. (2018b), including the type of SPME fiber [50-/30- $\mu \mathrm{m}$ divinylbenzene/ carboxen/polydimethylsiloxane (DVB/CAR/PDMS),

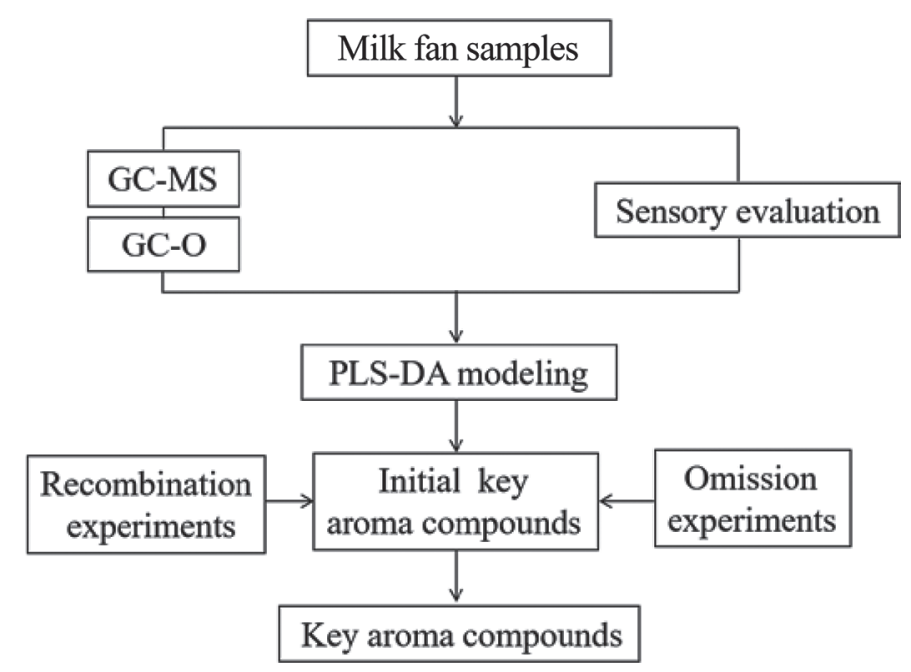

Figure 1. Process of identifying the key aroma compounds of milk fan. GC-O = gas chromatography-olfactometry; PLS-DA = partial least squares discriminant analysis. 
75- $\mu \mathrm{m}$ CAR/PDMS, and 65- $\mu \mathrm{m}$ PDMS/DVB], extraction temperature $\left(50,60,70\right.$, and $\left.80^{\circ} \mathrm{C}\right)$, and extraction time $(20,30,40$, and $50 \mathrm{~min})$. The sample vials were kept in a water bath at $60^{\circ} \mathrm{C}$ for $40 \mathrm{~min}$ for the GC-MS and GC-O analyses.

\section{GC-MS Analysis}

Volatile compounds were detected by an Agilent 7890-5973 GC-MS (Agilent Technologies, Santa Clara, CA) system and an HP-Innowax analytical fused-silica capillary column $(60 \mathrm{~m} \times 0.25 \mathrm{~mm} \times 0.25 \mu \mathrm{m}$; Agilent Technologies). The injection was in splitless mode. The oven temperature was held at $40^{\circ} \mathrm{C}$ for $4 \mathrm{~min}$, increased to $100^{\circ} \mathrm{C}$ at a rate of $3^{\circ} \mathrm{C} / \mathrm{min}$ and held for $2 \mathrm{~min}$, increased to $150^{\circ} \mathrm{C}$ at $4^{\circ} \mathrm{C} / \mathrm{min}$, and then increased to $230^{\circ} \mathrm{C}$ at $10^{\circ} \mathrm{C} / \mathrm{min}$ and maintained for $5 \mathrm{~min}$. Helium was used as the carrier gas at a constant flow rate of $1 \mathrm{~mL} / \mathrm{min}$. The electron ionization energy of the MS detector was $70 \mathrm{eV}$, the ion source temperature was $230^{\circ} \mathrm{C}$, with the quadrupole in scan mode (total ion currents from 30 to $450 \mathrm{~m} / z$ at $1 \mathrm{scan} / \mathrm{s}$ ).

The volatile compounds were identified by retention indices, which were determined by straight-chain alkanes $\left(\mathrm{C}_{6}-\mathrm{C}_{30}\right)$ and by comparing them with MS images in the NIST11 mass spectral database library. Volatile compounds were quantified using internal standards. Before quantification, an IS curve was prepared by adding $100 \mu \mathrm{L}$ of different concentrations of propionic acid and $100 \mu \mathrm{L}$ of IS solution $(13 \mathrm{mg} / \mathrm{L})$ to milk fan samples to confirm whether there was a linear relationship between the ratio of the concentration of the tested compounds $\left(\mathrm{C}_{\mathrm{i}}\right)$ to IS $\left(\mathrm{C}_{\mathrm{IS}}\right)\left(x=\mathrm{C}_{\mathrm{i}} / \mathrm{C}_{\mathrm{IS}}\right)$ and the ratio of their peak areas $(A)\left(y=A_{i} / A_{I S}\right)$ under certain chromatographic conditions. The standard curve was $y=0.7269 x+0.0004$ and the linear correlation factor $\mathrm{R}^{2}$ (0.993) of the standard curve met the experimental requirements. The formula for the relative content of volatile compounds is as follows: $C_{i}=f_{i} \times C_{I S} \times A_{i} / A_{I S}$, where $f_{i}=\left(A_{i} / A_{I S}\right) /\left(C_{i} / C_{I S}\right)$, representing the slope of the internal standard curve.

\section{GC-O Analysis}

Aroma compounds were identified using an Agilent 7890 GC system coupled with an olfactory detection port (Gerstel ODP-2, Mulheim an der Ruhr, Germany). The GC effluent was set to be separated equally between the flame-ionization detector and the sniffer. Milk fan samples were separated on an HP-Innowax $(60 \mathrm{~m} \times 0.25 \mathrm{~mm} \times 0.25 \mu \mathrm{m} ;$ Agilent Technologies $)$ analytical fused-silica capillary column.

The air carried the capillary column effluent into a glass funnel where odor-specific magnitude estimation
(OSME) analysis was conducted, which is a time-intensity method for GC-O. Fifteen panelists were trained before sniffing and were familiarized with the odor descriptions using solutions of the reference odorants. Panelists were required to note the onset and end time, odor characteristic, and intensity while sniffing the effluent from the sniffing mask. The aroma intensity was evaluated using a 5-point intensity scale from 0 to 5 , where 0 was none, 3 was medium, and 5 was extremely strong. Each experiment was performed in triplicate by each panelist. The aroma intensity value was the average of the 15 panelists.

\section{Odor Activity Values}

Odor activity values were used to evaluate the contribution of each compound to overall milk fan aroma, which was calculated as the ratio of the concentration of a single aroma compound to its detection threshold in air. The threshold values were taken from the literature.

\section{Sensory Evaluation}

The sensory evaluations, GC-O analysis, and aroma recombination and omission experiments were performed by 15 panelists ( 8 men and 7 women, average age $23 \mathrm{yr}$ ). All had previously received professional training in sensory evaluation. The odor descriptors (fruity, cheesy, milky, sour, rancid, musty, nutty, umami, sulfurous, and fatty) were chosen in preliminary tests by the panelists (Majcher et al., 2018). The 10 descriptors were defined as follows, and relevant material objects or chemical standards were used as references: $20 \mathrm{mg} / \mathrm{kg}$ ethyl hexanoate for "fruity"; fresh butter for "cheesy"; fresh milk for "milky"; $0.08 \%$ citric acid solution in water for "sour"; butanoic acid for "rancid"; potting soil for "musty"; unsalted raw nuts for "nutty"; $1 \%$ monosodium glutamate solution in water for "umami"; mashed boiled egg for "sulfurous"; and salad oil for "fatty" (Aday and Yuceer, 2014). The 8-g milk fan samples or recombinants were kept in an odorproof container $(100 \mathrm{~mL})$ until each panelist's evaluation. The odor properties were rated from 0 (none) to 10 (very strong). The tests were performed in triplicate.

\section{Aroma Recombination Experiments}

Aroma recombination was undertaken to further confirm the key aroma compounds. The 23 initial key aroma compounds with $\mathrm{OAV} \geq 1$ were dissolved in glycerol triacetate matrix in their natural concentrations (in Y6) and then equilibrated at an ambient temperature for $10 \mathrm{~min}$, and then a complete recombination 
model $\mathrm{Y}$ was obtained. A descriptive profile test was performed by the 15 panelists. The method was the same as described in the Sensory Evaluation section.

\section{Aroma Omission Experiments}

The contribution of aroma compounds to the overall aroma of milk fan was investigated by the triangle test, in which one or a group of compounds was omitted from the recombinant aroma model $\mathrm{Y}$ to obtain an omission model. Every omission model together with 2 recombinants ( $5 \mathrm{~g}$ each) was given to the panelists for sensory evaluation at random.

\section{Statistical Analysis}

Descriptive data and the differences between samples were analyzed by ANOVA and Duncan's test using SPSS (version 13.0; SPSS Inc., Chicago, IL). Then, PLS-DA was used to identify the contribution of the odor-active compounds to the sensory evaluation of milk fan by using $\mathrm{R}$ software with the mixOmics package (http://mixomics.org). Partial least squares discriminant analysis applies a quantitative partial least squares method to discriminant analysis and is suitable for multidimensional data analysis.

\section{RESULTS AND DISCUSSION}

\section{Optimization of Headspace-SPME Conditions}

Three headspace-SPME variables (type of SPME fiber, extraction temperature, and extraction time) were investigated. The efficiency of the extraction process is critically dependent on the fiber coating (Risticevic et al., 2010). The highest number of aroma compounds and highest total peak areas were obtained with the $75-\mu \mathrm{m}$ CAR/PDMS fiber. The polarity of this coating material is more consistent with the volatile compounds in milk fan and has a larger partition coefficient, so the extraction and flavor enrichment ability of the milk fan is stronger (Lucey, 2011). In general, the number of adsorbed compounds increases sharply at the beginning of the extraction process, and the number of components tends to stabilize after reaching a certain equilibrium point. Extraction time strongly influences the extraction effect. The results showed that the number of aroma compounds and the total peak areas obtained after 40 min of extraction were greater than those obtained after $50 \mathrm{~min}$, indicating that extraction reached equilibrium after $40 \mathrm{~min}$. If the extraction time is too long, the accuracy of the relative content of the analyte will be reduced because the compounds compete for position on the fiber. If the fiber stays in the headspace too long, this competition can cause inaccuracies in the relative amounts of analytes (Howard et al., 2005). Extraction temperature also has a strong effect on the type and quantity of volatile compounds in the samples (Rocha et al., 2001). The results showed that the number of aroma compounds and the total peak areas were maximized when the extraction temperature reached 50 to $60^{\circ} \mathrm{C}$, and then remained steady as the temperature increased to $80^{\circ} \mathrm{C}$. The aroma extraction of milk fan was therefore carried out with the $75-\mu \mathrm{m}$ CAR/PDMS SPME fiber at $60^{\circ} \mathrm{C}$ with an extraction time of $40 \mathrm{~min}$.

\section{Quantitative Analysis of Aroma Compounds of Milk Fan}

Seventy-one aroma compounds were detected, including 10 acids, 8 aldehydes, 9 alcohols, 9 ketones, 19 esters, 11 aromatic and heterocyclic compounds, and 5 other compounds. Acids (30.8-71.6\%; percentages indicate the ratio of the content of this substance to the total aroma content), esters (3.94-67.6\%), and ketones (2.73-17.9\%) were the most abundant groups. The main aroma compounds analyzed by GC-MS were consistent with other types of cheeses analyzed by this method, including Cheddar, Camembert, Grana Padano, and Gruyère (Curioni and Bosset, 2002).

Acids were the compounds present at the highest levels. The fatty acid content in milk fan (total acids $\sim 9,400$ to $21,100 \mu \mathrm{g} / \mathrm{kg}$ ) was significantly higher than that in rennet-curd cheese (total acids $3,028 \mu \mathrm{g} / \mathrm{kg}$; (Hayaloglu et al., 2013). The high fatty acid content may be due to the unique acidulation processing technology of milk fan. Unlike a curd-coagulated cheese, milk fan is a traditional acid-curd cheese made by adding papaya acid juice to the raw milk (Zhang et al., 2014). The acid juice is also known as whey. It contains many probiotic strains and normally contains Lactobacillus acidophilus to promote acid development (Gallardo-Escamilla et al., 2005). The butanoic acid content of the milk fan $(3,190-10,700 \mu \mathrm{g} / \mathrm{kg})$ was higher than that of Gokceada cheese $(677 \mu \mathrm{g} / \mathrm{kg})$, as was the hexanoic acid content (milk fan: 2,900-6,500 $\mu \mathrm{g} / \mathrm{kg}$; Gokceada: $820 \mu \mathrm{g} / \mathrm{kg}$ ) and octanoic acid content (milk fan: 1,400-2,800 $\mu \mathrm{g} /$ kg; Gokceada: $305 \mu \mathrm{g} / \mathrm{kg}$ ). From a biochemical point of view, electrophoretic patterns have demonstrated that $\beta-\mathrm{LG}$ is the main protein present in ricotta forte (a type of acid-curd cheese), indicating that intermediate levels of proteolysis took place in acid-curd cheese. The main biochemical event was lipolysis and many short-chain fatty acids were produced (Faccia et al., 2018). However, some of the acids (propanoic and butanoic) in milk fan in the current study were present at levels lower than in Cheddar (Drake et al., 2010). Fatty acids play an important role in the aroma of milk 
fan. Butanoic acid $(3,190-10,700 \mu \mathrm{g} / \mathrm{kg})$, hexanoic acid $(2,900-6,500 \mu \mathrm{g} / \mathrm{kg})$, octanoic acid $(1,400-2,800 \mu \mathrm{g} /$ $\mathrm{kg})$, and decanoic acid $(340-600 \mu \mathrm{g} / \mathrm{kg})$ were the most abundant acids in the 6 milk fan samples. Butanoic acid has a rancid cheese-like odor that plays an important role in the flavor in many cheeses, such as Camembert, Cheddar, Grana Padano, and Gruyère. Hexanoic acid is also a characteristic flavor component of Grana Padano and Roncal. Octanoic acid and decanoic acids are the main odorants of the latter cheese (Curioni and Bosset, 2002).

Other major volatile groups were esters and ketones. A total of 19 esters were detected in the milk fan, the most abundant of which were ethyl acetate, ethyl butyrate, ethyl hexanoate, ethyl caprylate, and ethyl caprate. It is well known that esters are important contributors to the flavor of cheese. Based on a low threshold, they were capable of masking some bad odors caused by large amounts of short-chain free amino acids, methyl ketones, and amines (Holland et al., 2005). Ethyl acetate, ethyl butyrate, and ethyl hexanoate play important roles in the formation of the fruity properties of Parmigiano-Reggiano, Cheddar, and other cheeses (Fenster et al., 2003). The ethyl acetate content of the milk fan $(210-1,220 \mu \mathrm{g} / \mathrm{kg})$ was higher than that of Parmigiano-Reggiano $(50 \sim 250 \mu \mathrm{g} /$ $\mathrm{kg})$. Ethyl butyrate and ethyl hexanoate (300-3,500 $\mu \mathrm{g} / \mathrm{kg}$ ) are considered key aroma compounds of cheese. The ethyl butyrate content of the milk fan (500-7,000 $\mu \mathrm{g} / \mathrm{kg})$ was higher than that of Mozzarella $(700 \mu \mathrm{g} /$ $\mathrm{kg}$ ) and Emmental $(260-500 \mu \mathrm{g} / \mathrm{kg}$ ) (Liu et al., 2004). Other ester compounds may help to produce a very mild sweet background aroma (Majcher et al., 2014). Ketones are a rich constituent of many dairy products and have characteristic odors and low perception thresholds (Frank et al., 2004). Nine ketones (mostly methyl ketones) were detected in the milk fan, with 2-pentanone, 2-heptanone, 2-octanone, and 2-nonanone being the most abundant. The main sources of flavor in raw and semi-ripe cheeses are the corresponding volatile constituents of methyl ketones, which are produced by $\beta$-oxidation of acyl lipids (Matera et al., 2018). Fatty acids are the substrate for ester and methyl ketone formation (Law and Wigmore, 2007). Because of the high content of free fatty acids in milk fan, the contents of ketones and esters were also relatively high (Table 1).

Fruity, floral, and musty notes are associated with various methyl ketones, such as 2-octanone, 2-heptanone, 2-nonanone, and 2-undecanone. The herbal odor of 2-heptanone may play an important role in the formation of the fruit properties of milk fan, consistent with Emmental and Gorgonzola cheeses. These aroma compounds are believed to have a positive effect on the aroma of cheese (Lee et al., 2003).

\section{Odor-Active Compounds Identified by GC-O}

Gas-chromatography olfactometry analysis is an effective means of identifying odor-active compounds in milk fan. The olfactometry results are provided in Table 2. Thirty-one aroma compounds were detected in total. The aroma intensities of the compounds ranged from 1.00 to 4.89. Compounds with a medium or strong aroma intensity of $\geq 3$, which are likely to contribute to the aroma characteristics of milk fan, included propionic acid, butyric acid, pentanoic acid, hexanoic acid, heptanoic acid, octanoic acid, hexanal, pentanol, hexanol, 1-octen-3-ol, 2-pentanone, ethyl butyrate, ethyl hexanoate, ethyl caprylate, and ethyl caprate. Acids were the most abundant compounds in milk fan. Eight acids were identified (Table 2): propanoic acid (aroma intensity: 1.18-4.51), butanoic acid (4.10-4.82), pentanoic acid (1.31-3.86), hexanoic acid (1.76-4.62), heptanoic acid (1.25-3.50), octanoic acid (1.50-4.73), nonanoic acid (1.55), and $n$-decanoic acid (1.39-2.70). The panelists' sensory descriptions suggested that these acids generally contributed "cheese," "rancid," "fat," and "soy" notes. These acids are widely found by GC-O in many other cheeses (Liu et al., 2004). Short- and medium-chain fatty acids $\left(\mathrm{C}_{4}-\mathrm{C}_{12}\right)$ have low perception thresholds and provide typical aroma notes to cheeses such as Cheddar, Gruyère, Roncal, Emmental, Camembert, and Grana Padano (Curioni and Bosset, 2002). These compounds were the main acids in milk fan, which has strong and rich flavors of milk and acid decay.

Aldehydes, esters, and ketones were also found to be important odor-active compounds of milk fan. Isovaleraldehyde, hexanal, octanal, (E)-2-heptenal, nonanal, (E)-2-octenal, 2-pentanone, 2-heptanone, 3-octanone, 2-nonanone, ethyl acetate, ethyl butyrate, ethyl valerate, and ethyl hexanoate were the odor-active compounds of milk fan. In addition to (E)-2-heptenal, (E)2-octenal, and ethyl valerate, these compounds were also odor-active compounds in Cheddar, Mozzarella, Grana Padano, Ragusano, Emmental, and Gorgonzola cheeses (Curioni and Bosset, 2002).

\section{Correlation of Odor-Active Compounds with Sensory Characteristics of Milk Fan}

The relationships between the 31 odor-active compounds and the 10 sensory evaluation properties were explored using a PLS-DA algorithm to investigate the effect and contribution of compounds on the sensory characteristics of milk fan. The statistical results of the sensory profile analysis of the 10 odor properties are shown in Figure 2. It is well known that factors such as geography, climate, and cattle diet affect the chemical 


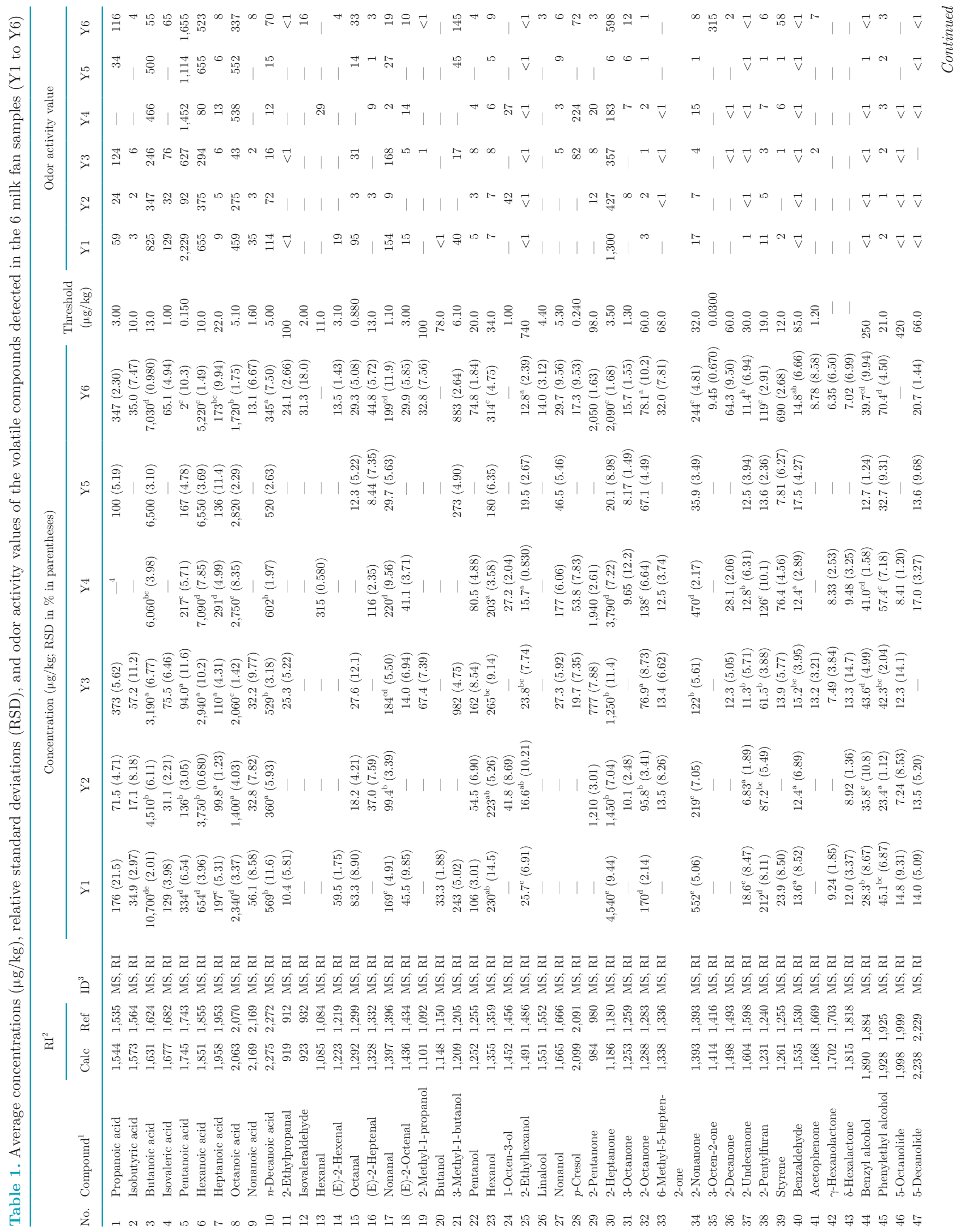


IDENTIFYING KEY AROMA COMPOUNDS IN CHINESE MILK FAN

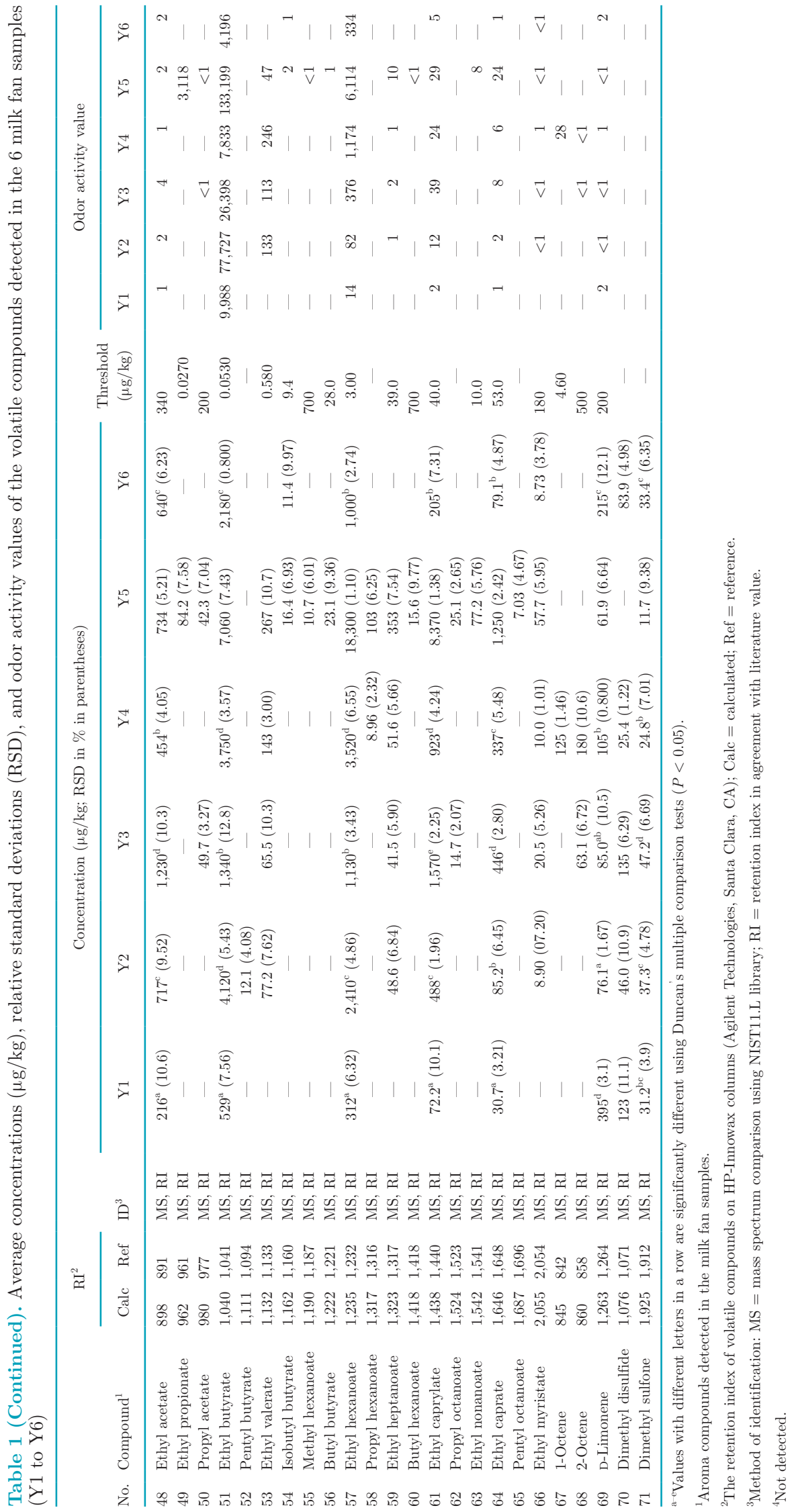




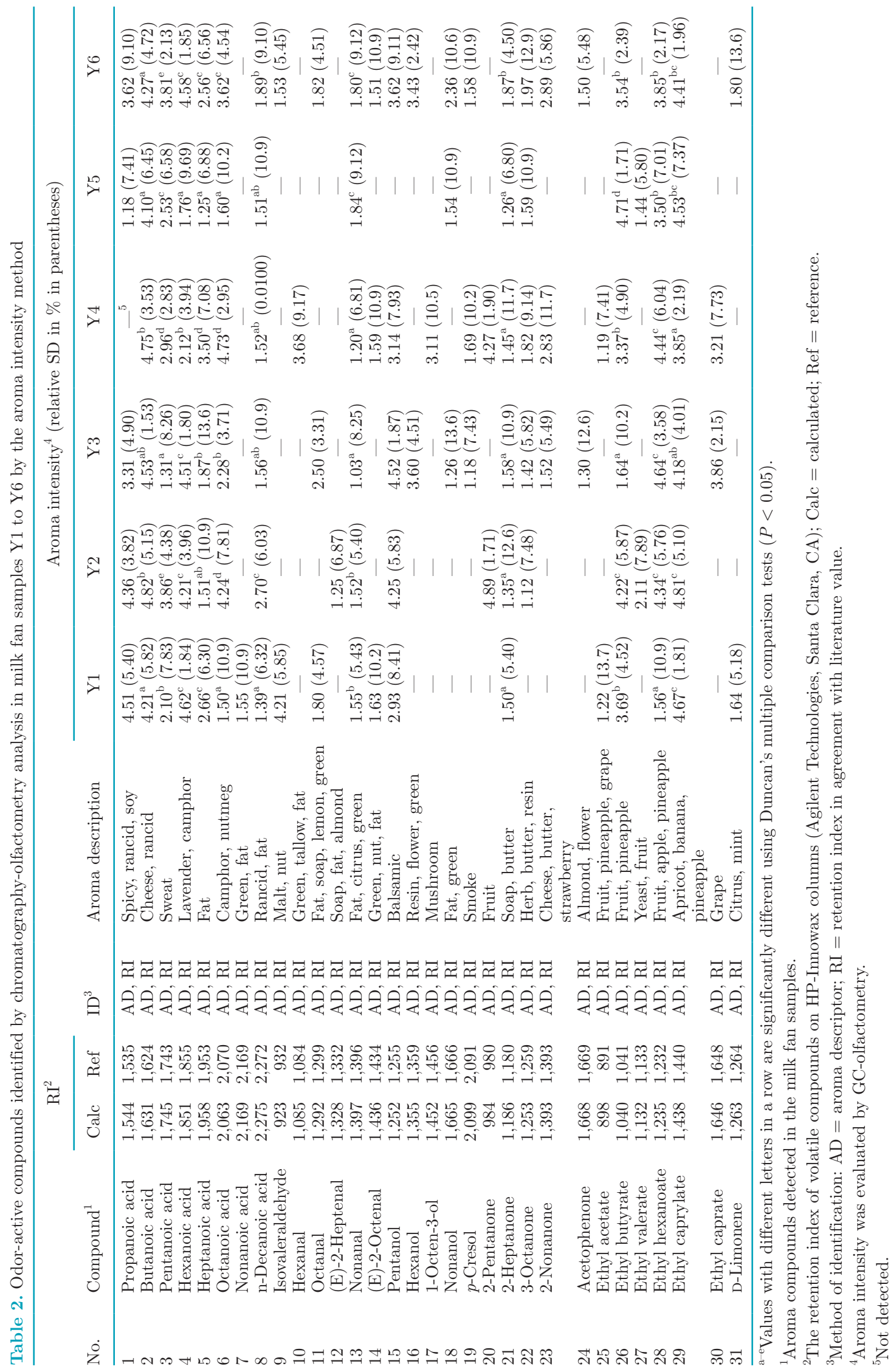


composition of milk, which in turn affects the sensory quality of milk and cheese (Carpino et al., 2004; Rapisarda et al., 2013). Based on the Dengchuan region's particular geographical climate, rich vegetation, and breed of dairy cattle, the milk from this area has a strong flavor and high fat content, which give milk fan its rich and unique flavor. The prominent aromas of the milk fan are cheesy, milky, sour, fruit, fatty, and rancid. The overall aroma is typically mild. Some pungent odors, such as sulfur and musty, are not obvious. In general, acid and acid- or heat-coagulated cheeses have a mild or acidic aroma. They are usually fresh cheeses. The "sour" and "rancid" descriptors were significantly different between samples $(P<0.05)$, which might be related to the processing technology of milk fan and the amount of papaya acid juice added.

Twenty-three of the pairwise associations between the odor-active compounds and the sensory evaluations were significant (Figure 3). Nonanoic acid was positively correlated with nutty properties; octanoic acid and propanoic acid were positively correlated with cheesy properties; pentanoic acid was negatively correlated with sulfurous properties; butanoic acid, 2-nonanone, and 2-heptanone were positively correlated with rancid properties; nonanol was positively correlated with fatty properties and negatively correlated with fruity properties; octanal was positively correlated with fatty and nutty properties; (E)-2-octenal was positively correlated with rancid properties and negatively correlated with cheesy and sulfurous properties; ethyl caprylate and ethyl hexanoate were positively correlated with fruity properties; ethyl butyrate was positively correlated with cheesy and fruity properties and negatively

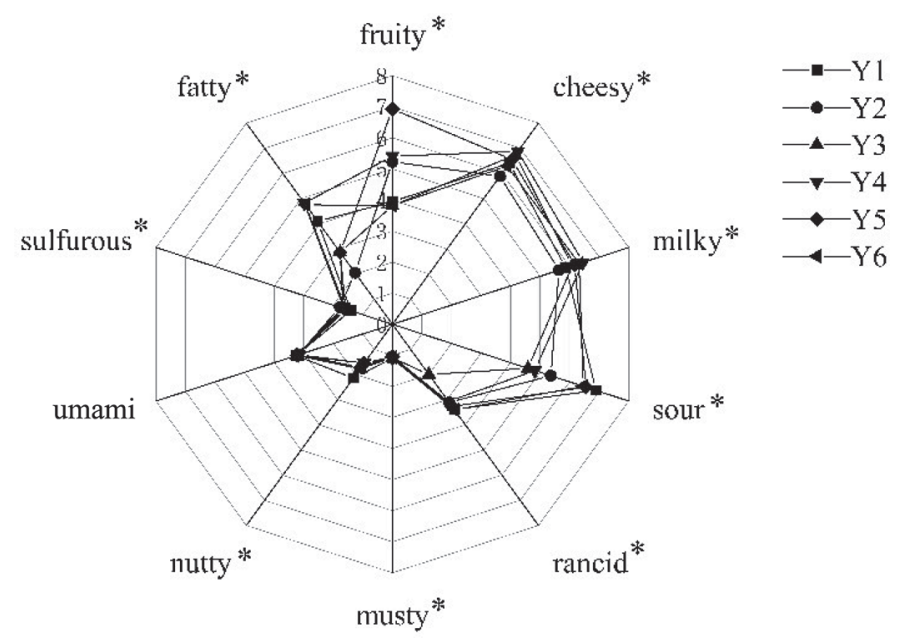

Figure 2. Aroma profiles of milk fan obtained from the Y1, Y2, Y3, Y4, Y5, and Y6 samples. The asterisks indicate that values are significantly different using Duncan's multiple comparison tests $(P<$ $0.05)$. correlated with nutty properties; ethyl valerate was positively correlated with fruity properties and negatively correlated with nutty properties; and D-limonene was positively correlated with rancid properties and negatively correlated with cheesy properties. Hence, these compounds (nonanoic acid, octanoic acid, propanoic acid, pentanoic acid, butanoic acid, 2-nonanone, 2-heptanone, nonanol, octanal, (E)-2-octenal, ethyl caprylate, ethyl hexanoate, ethyl butyrate, ethyl valerate, and D-limonene) seem to be the initial key aroma compounds of milk fan and may contribute to the overall aroma of the cheese.

\section{Aroma Recombination and Omission Experiments}

An aroma recombination experiment was performed to provide further information about the initial key aroma compounds. Omission experiments were performed to evaluate the contribution of the initial key aroma compounds to the overall aroma of milk fan and to identify the key aroma compounds of milk fan. Figure 4 shows that the aroma profile of the recombinant was similar to that of the original milk fan, sample Y6. The sensory properties (fruity, cheesy, milky, sour, rancid, musty, nutty, umami, sulfurous, and fatty) were generally consistent with those of Y6. Only 2 properties (fruity and rancid) showed a slight but nonsignificant difference $(P>0.05)$, suggesting a higher amount of the corresponding aroma compounds. The results showed that the typical aroma of milk fan was successfully simulated by combining 23 initial odor-active compounds (at their natural concentrations in Y6).

In the omission experiment, 23 aroma omission models were studied. The data (Table 3) suggested that all panelists could detect the missing group of acids in model 1 , with a highly significant $(P \leq 0.001)$ difference between the omission model and the recombination model. This indicates that these fatty acid odors play a key role in the overall aroma of milk fan. Furthermore, models 1-1, 1-2, and 1-6 (lacking propanoic acid, butanoic acid, and octanoic acid, respectively) were significantly different $(P \leq 0.001)$ from the recombination model. Therefore, propanoic acid, butanoic acid, and octanoic acid might be important contributors to the aroma of milk fan. Similar results were found for models $2,2-2,2-3,4,4-2,5$, and $5-2$, indicating that octanal, nonanal, 2-nonanone, and ethyl hexanoate might also play a key role in the aroma. These results provide further evidence that these are indeed the key aroma compounds in milk fan, confirming the GC-O results. Pentanoic acid (OAV of 92-2,230), hexanoic acid (OAV of 80-655), heptanoic acid (OAV of 5-13), and decanoic acid (OAV of 12-114) had high OAV (Table 2). However, in the triangle tests, only $6,3,5$, 


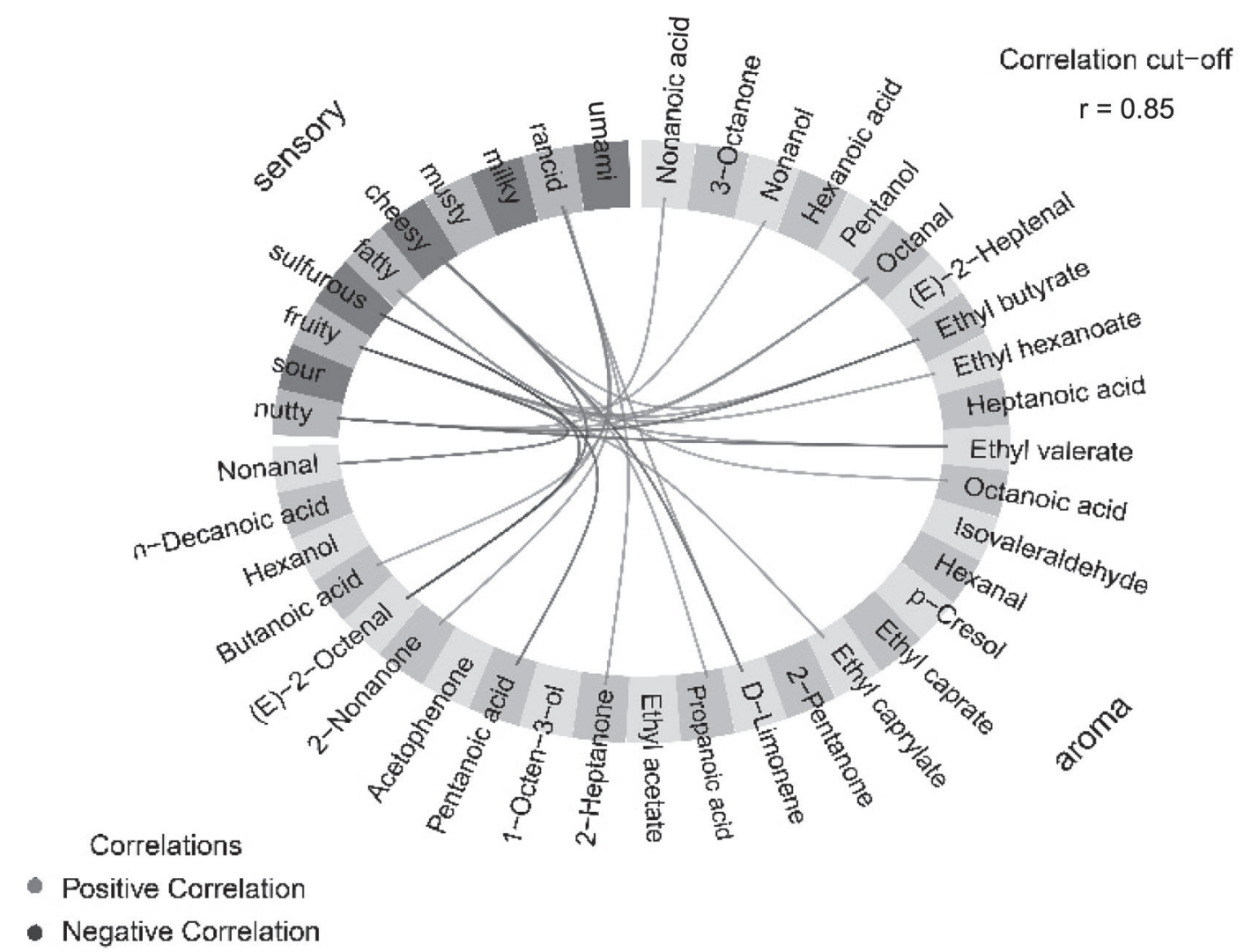

Figure 3. Correlations between odor-active components and sensory properties of 6 milk fan samples by partial least squares discriminant analysis. Gray lines in the circle represent positive correlations and black lines represent negative correlations between odor-active components and sensory properties. Ten sensory odor properties and 31 odor-active components are shown, as listed in Table 2.

and 6 of the 15 panelists $(P>0.05)$ were correct in detecting the omission models $1-3,1-4,1-5$, and $1-7$, respectively. These 4 compounds were thus not considered to be key aroma contributors. Similar results were

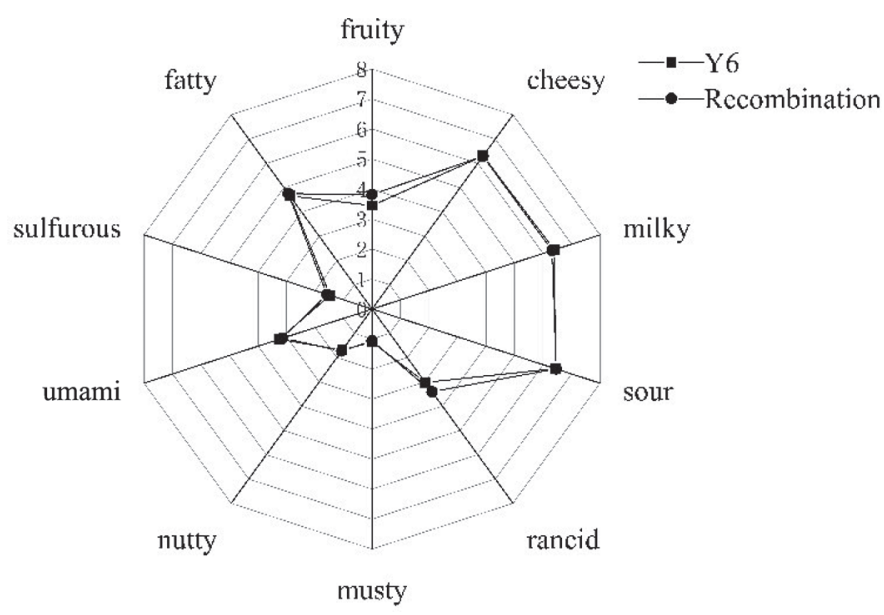

Figure 4. Aroma profile of the Y6 sample of milk fan and the complete aroma recombination. found for models $2-1,2-4,3,4-1,4-3,5-1,5-3,6$, and 7, which were missing isovaleraldehyde, (E)-2-octenal, pentanol, hexanol, nonanol, 2-heptanone, 3-octanone, ethyl butyrate, ethyl caprylate, acetophenone, $p$-cresol, and D-limonene, respectively, indicating that these compounds had no significant effect on the aroma of milk fan.

In summary, the key aroma profile of milk fan consisted of propanoic acid, butanoic acid, octanoic acid, octanal, nonanal, 2-nonanone, and ethyl hexanoate. Propanoic acid and butanoic acid contribute to the cheese and rancid aromas, octanal and nonanal contribute to the fat aroma, octanoic acid and 2-nonanone contribute to the cheese aroma, and ethyl hexanoate contributes to the fruity aroma.

\section{CONCLUSIONS}

The aroma characteristics of 6 milk fan cheeses were studied using GC-MS, GC-O, sensory evaluation, PLS$\mathrm{DA}$, and aroma recombination and omission experiments. Seventy-one aroma compounds were detected by GC-MS, and 31 were identified as odor-active com- 
Table 3. Omission experiments from the complete recombination model

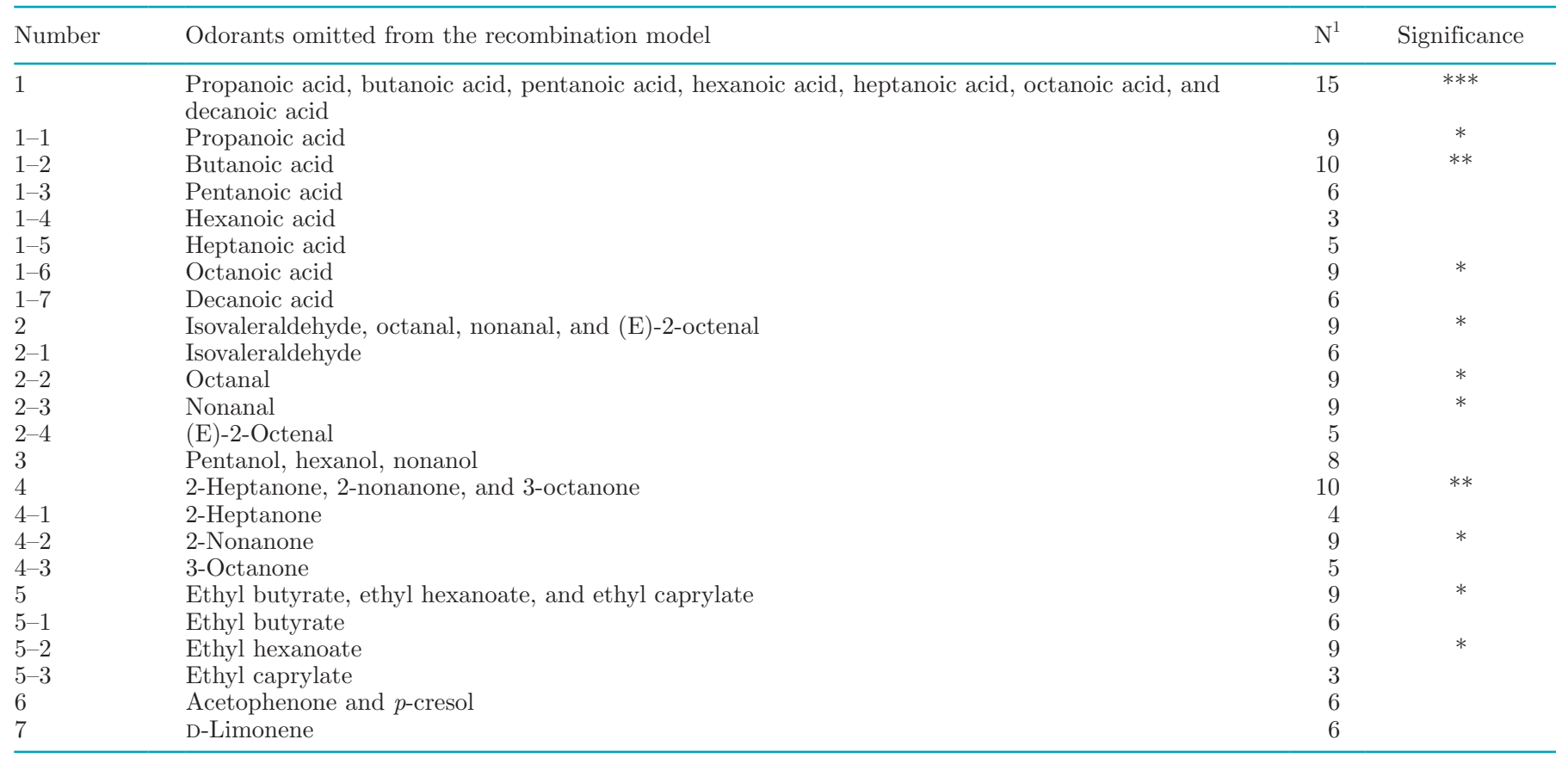

${ }^{1}$ Number of correct judgments from 15 panelists evaluating the aroma difference by means of a triangle test.

$* * * P \leq 0.001,{ }^{* *} P \leq 0.01,{ }^{*} P \leq 0.05$.

pounds by GC-O. The PLS-DA results showed that the initial key aroma compounds had good correlations with sensory properties. Based on the aroma recombination and omission experiments, 7 odor-active compounds were further identified as the key aroma compounds: 3 acids (propanoic, butanoic, and octanoic), 2 aldehydes (octanal and nonanal), and 2-nonanone and ethyl hexanoate. In future work, nonvolatile flavor compounds should be analyzed to better understand the flavor characteristics of milk fan.

\section{ACKNOWLEDGMENTS}

This work was sponsored by the National Natural Science Foundation of China (Award No. 31771943), the "Shu Guang" project (No. 16SG50) and Shanghai Rising-Star Program (No. 17QB1404200) supported by Shanghai Municipal Education Commission and the Shanghai Education Development Foundation for financial support.

\section{REFERENCES}

Aday, S., and Y. K. Yuceer. 2014. Physicochemical and sensory properties of Mihalic cheese. Int. J. Food Prop. 17:2207-2227.

Bertuzzi, A. S., P. L. H. McSweeney, M. C. Rea, and K. N. Kilcawley. 2018. Detection of volatile compounds of cheese and their contribution to the flavor profile of surface-ripened cheese. Compr. Rev. Food Sci. Food Saf. 17:371-390.
Carpino, S., J. Horne, C. Melilli, G. Licitra, D. M. Barbano, and P. J. Soest. 2004. Contribution of native pasture to the sensory properties of Ragusano cheese. J. Dairy Sci. 87:308-315.

Curioni, P. M. G., and J. O. Bosset. 2002. Key odorants in various cheese types as determined by gas chromatography-olfactometry. Int. Dairy J. 12:959-984.

Drake, M. A.. R. E. Miracle, and D. J. McMahon. 2010. Impact of fat reduction on flavor and flavor chemistry of Cheddar cheeses. J. Dairy Sci. 93:5069-5081.

Dunkel, A., M. Steinhaus, M. Kotthoff, M. Nowak, B. Krautwurst, and P. Schieberle. 2014. Nature's chemical signatures in human olfaction: A foodborne perspective for future biotechnology. Angew. Chem. Int. Ed. Engl. 53:7124-7143.

Faccia, M., A. Trani, G. Natrella, and G. Gambacorta. 2018. Short communication: Chemical-sensory and volatile compound characterization of ricotta forte, a traditional fermented whey cheese. J. Dairy Sci. 101:5751-5757.

Fenster, K. M., S. A. Rankin, and J. L. Steele. 2003. Accumulation of short n-chain ethyl esters by esterases of lactic acid bacteria under conditions simulating ripening Parmesan cheese. J. Dairy Sci. 86:2818-2825.

Fox, P. F., and J. M. Wallace. 1997. Formation of flavor compounds in cheese. Adv. Appl. Microbiol. 45:17-85.

Frank, D. C., C. M. Owen, and J. Patterson. 2004. Solid-phase microextraction (SPME) combined with gas-chromatography and olfactometry-mass spectrometry for characterization of cheese aroma compounds. Lebensm. Wiss. Technol. 37:139-154.

Gallardo-Escamilla, F. J., A. L. Kelly, and C. M. Delahunty. 2005. Influence of starter culture on flavor and headspace volatile profiles of fermented whey and whey produced from fermented milk. J. Dairy Sci. 88:3745-3753

Gao, W., W. Fan, and Y. Xu. 2014. Characterization of the key odorants in light aroma type Chinese liquor by gas chromatography-olfactometry, quantitative measurements, aroma recombination, and omission studies. J. Agric. Food Chem. 62:5796-5804.

Gracka, A., H. H. Jeleń, M. Majcher, A. Siger, and A. Kaczmarek. 2016. Flavoromics approach in monitoring changes in volatile com- 
pounds of virgin rapeseed oil caused by seed roasting. J. Chromatogr. A 1428:292-304.

Hayaloglu, A. A., K. Yasar, C. Tolu, and D. Sahingil. 2013. Characterizing volatile compounds and proteolysis in Gokceada artisanal goat cheese. Small Rumin. Res. 113:187-194.

Holland, R., S. Q. Liu, V. L. Crow, M. L. Delabre, M. Lubbers, M. Bennett, and G. Norris. 2005. Esterases of lactic acid bacteria and cheese flavour: Milk fat hydrolysis, alcoholysis and esterification. Int. Dairy J. 15:711-718.

Howard, K. L., J. H. Mike, and R. Riesen. 2005. Validation of a solid-phase microextraction method for headspace analysis of wine aroma components. Am. J. Enol. Vitic. 56:37-45.

Jo, Y., D. M. Benoist, A. Ameerally, and M. A. Drake. 2018a. Sensory and chemical properties of Gouda cheese. J. Dairy Sci. 101:19671989.

Jo, Y., D. M. Benoist, D. M. Barbano, and M. A. Drake. 2018b. Flavor and flavor chemistry differences among milks processed by high temperature, short time or ultra-pasteurization. J. Dairy Sci. 101:3812-3828.

Karametsi, K., S. Kokkinidou, I. Ronningen, and D. G. Peterson. 2014. Identification of bitter peptides in aged cheddar cheese. J. Agric. Food Chem. 62:8034-8041.

Law, B. A., and A. S. Wigmore. 2007. Effect of commercial lipolytic enzymes on flavor development in Cheddar cheese. Int. J. Dairy Technol. 38:86-88.

Lee, J. H., R. Diono, G. Y. Kim, and D. B. Min. 2003. Optimization of solid phase microextraction analysis for the headspace volatile compounds of parmesan cheese. J. Agric. Food Chem. 51:11361140.

Liu, S. Q., R. Holland, and V. L. Crow. 2004. Esters and their biosynthesis in fermented dairy products: A review. Int. Dairy J. 14:923-945.

Liu, W., Z. H. Sun, J. C. Zhang, W. Gao, W. H. Wang, L. Wu, T. S. Sun, W. Chen, X. M. Liu, and H. P. Zhang. 2009. Analysis of microbial composition in acid whey for dairy fan making in Yunnan by conventional method and 16S rRNA sequencing. Curr. Microbiol. 59:199-205.

Liu, W. J., H. Y. Li, Y. F. Chen, W. Gao, X. Chen, X. M. Liu, and H. P. Zhang. 2011. Analysis of chemical composition, protein profile and microstructure of dairy fan in Yunnan Province of China. Milchwissenschaft 66:57-60.

Lucey, J. A. 2011. Acid and acid/heat coagulated cheese. Pages 698-705 in Cheese: Encyclopedia of Dairy Sciences. 2nd ed. J. W. Fuquay, ed. Academic Press. London, UK.

Ma, H., L. Oxley, A. Rae, C. Fan, J. Huang, and S. Rozelle. 2012. The evolution of productivity performance on China's dairy farms in the new millennium. J. Dairy Sci. 95:7074-7085.

Majcher, M. A., K. Myszka, A. Grygier, and G. Anna. 2018. Key odorants of Lazur-A Polish type mold-ripened cheese. J. Agric. Food Chem. 66:2443-2448.

Majcher, M. A., K. Myszka, J. Kubiak, and H. H. Jelen. 2014. Identification of key odorants of fried cottage cheese and contribution of Galactomyces geotrichum MK017 to the formation of 2-phenyl- ethanol and related rose-like aroma compounds. Int. Dairy J. 39:324-329.

Masotti, F., S. Cattaneo, M. Stuknyte, G. Batteli, L. Vallone, and I. De Noni. 2017. Composition, proteolysis, and volatile profile of Strachitunt cheese. J. Dairy Sci. 100:1679-1687.

Matera, J., A. S. Luna, D. B. Batista, T. C. Pimentel, J. Moraes, B. A. Kamimura, M. V. Ferreira, H. L. A. Silva, S. P. Mathias, E. A. Esmerino, M. Q. Freitas, R. S. L. Raices, S. L. Quitério, A. S. Sant'Ana, M. C. Silva, and A. G. Cruz. 2018. Brazilian cheeses: A survey covering physicochemical characteristics, mineral content, fatty acid profile and volatile compounds. Food Res. Int. 108:18-26.

Medvedev, V., O. Kurasova, J. Bernatavičienè, P. Treigys, V. Marcinkevičius, and G. Dzemyda. 2017. A new web-based solution for modelling data mining processes. Simul. Model. Pract. Theory $76: 34-46$.

Pelin, S., G. Gokce, K. Perihan, B. Deniz, and E. Zafer. 2018. Composition, proteolysis, lipolysis, volatile compound profile and sensory characteristics of ripened white cheeses manufactured in different geographical regions of Turkey. Int. Dairy J. 87:26-36.

Rapisarda, T., C. Pasta, G. Belvedere, and I. Schadt. 2013. Variability of volatile profiles in milk from the PDO Ragusano cheese production zone. Dairy Sci. Technol. 93:117-134.

Risticevic, S., H. Lord, T. Górecki, C. L. Arthur, and J. Pawliszyn 2010. Protocol for solid-phase microextraction method development. Nat. Protoc. 5:122-139.

Rocha, S., V. Ramalheira, A. Barros, I. Delgadillo, and M. A. Coimbra. 2001. Headspace solid phase microextraction (SPME) analysis of flavor compounds in wines: Effect of the matrix volatile composition in the relative response factors in a wine model. J. Agric. Food Chem. 49:5142-5151.

Song, H., and J. Liu. 2018. GC-O-MS technique and its applications in food flavor analysis. Food Res. Int. 114:187-198.

Zabaleta, L., K. Gourrat, L. J. R. Barron, M. Albisu, and E. Guichard. 2016. Identification of odour-active compounds in ewes' raw milk commercial cheeses with sensory defects. Int. Dairy J. 58:23-30.

Zhang, H. P., X. Chen, T. Dan, and J. Tong. 2014. Traditional Chinese fermented dairy foods. Pages 493-535 in Lactic Acid Bacteria. Springer, Amsterdam, the Netherlands.

Zhang, M., X. Chen, K. Hayat, E. Duhoranimana, X. M. Zhang, S. Q. Xia, J. Y. Yu, and F. L. Xing. 2018. Characterization of odoractive compounds of chicken broth and improved flavor by thermal modulation in electrical stewpots. Food Res. Int. 109:72-81.

Zhao, D., D. M. Shi, J. Y. Sun, A. J. Li, B. G. Sun, M. M. Zhao, F. Chen, X. T. Sun, H. H. Li, M. Q. Huang, and F. P. Zheng. 2018. Characterization of key aroma compounds in Gujinggong Chinese Baijiu by gas chromatography-olfactometry, quantitative measurements, and sensory evaluation. Food Res. Int. 105:616-627.

Zhu, J., and Z. Xiao. 2018. Characterization of the major odor-active compounds in dry Jujubes cultivars by application of gas chromatography-olfactometry and odor activity value. J. Agric. Food Chem. 66:7722-7734 